\title{
Los marcadores discursivos en las obras lexicográficas ${ }^{1}$
}

Nancy Vázquez Veiga Universidade da Coruña

\section{INTRODUCCIÓN}

Los estudios sobre marcadores discursivos dentro del ámbito hispano suelen empezar señalando que la tradición gramatical española apenas se ha ocupado de estos elementos. En realidad, las gramáticas casi no los mencionan, y únicamente en las obras lexicográficas podemos encontrar alguna referencia más o menos útil sobre ellos. No obstante, la información que nos proporciona este tipo de obras concierne básicamente al significado, dejando de lado otros aspectos (sintácticos, pragmáticos, etc.) que tienen gran importancia a la hora de caracterizar los marcadores.

Cuando se trata de definir un marcador discursivo, los diccionarios de lengua se decantan a menudo por las definiciones sinonímicas. Al presentar el significado de un marcador, el diccionario no hace alusión a sus posibles sentidos, sino que nos remite a otra partícula o a un conjunto de ellas. Tampoco resultan claras y precisas las definiciones perifrásticas que algunas obras lexicográficas ofrecen de estos elementos. Si, como señala J. FERnÁndez SEVILLA, la definición que un diccionario proporciona sobre una forma "debe resultar suficiente para informar acerca del valor, contenido y posibilidades combinatorias de la entrada, de la cual serán equivalentes" (1974, p. 70), podemos decir que, en conjunto, el tratamiento que se da a los marcadores discursivos en estas obras deja mucho que desear. Esta crítica la podemos extender a los diccionarios de sinónimos, en los que la confusión es todavía mayor.

1 Este artículo está basado en una comunicación que, con el título de "Marcadores discursivos: una aproximación al problema de la sinonimia". presenté en el XXIV Simposio de la Sociedad Española de Língüística (diciembre, 1994). Me complace expresar mi profundo agradecimiento a los profesores M. Alonso Ramos y M. Fernández Rodríguez, por las sugerencias que me han hecho durante la elaboración de este trabajo. 
En este artículo nos centraremos principalmente en aquellas definiciones sinonímicas que figuran en algunas obras lexicográficas a propósito de un grupo determinado de marcadores. Al margen de la conveniencia o no de este tipo de definiciones, se podrían considerar válidas, siempre y cuando nos encontremos ante "verdaderos" sinónimos. Pero en este caso tenemos un problema añadido, y es que, en general, no resulta sencillo decidir si dos voces son o no sinónimas. Incluso el vocablo sinonimia es bastante impreciso como término lingüístico ${ }^{2}$. Dependiendo del punto de partida que se adopte, dependiendo de cómo interpretemos este concepto, los resultados a los que se llegue serán diferentes. Según S. GuTIÉRREZ ORDóNEZ:

Las teorías configuran un espectro que va desde las que niegan furibundamente su existencia hasta las que la defienden paladinamente, pasando por todos los grados intermedios imaginables. La causa de tal diversidad hay que achacarla a varios hechos: diferente concepción de la sinonimia (total-parcial, estricta-laxa), distinto concepto de significado, aceptación o rechazo de la connotación, niveles del lenguaje, solución ofrecida al problema de la homonimia, el contexto, etc. (1989, p. 117).

A nuestro juicio hay dos aspectos fundamentales que debemos tener en cuenta al hablar de los posibles sinónimos de un marcador. Por un lado, no podemos establecer el análisis de la sinonimia a nivel de palabras, sino a nivel de los sentidos que tiene cada palabra. Por consiguiente, es absurdo decir que dos marcadores son sinónimos entre sí. Por otro lado, debemos ser consecuentes con la nueva dimensión que adquiere esta relación léxica cuando, como en este caso, nos situamos en el nivel del discurso ${ }^{3}$.

Este trabajo se estructura en tres partes. En primer lugar, observaremos lo que dicen algunas obras lexicográficas sobre los marcadores seleccionados. Se trata de formas que pueden desempeñar las funciones textuales de "resumen", "conclusión" y "cierre"4: en resumen, en resumidas cuentas, en suma, total, en conclusión, en fin, por último, fi-

2 C. OtAOLA Olano subraya la importancia que tiene el concepto de sinonimia en los trabajos lexicográficos:

La incidencia de la sinonimia en la confección de diccionarios tiene una doble vertiente. Por una parte, la confección de diccionarios de sinónimos, y, por otra, el empleo de la sinonimia como forma de definición de los artículos de un diccionario (1994, p. 137).

3 Richard Patry y Nathan MénaRd señalan:

La notion de synonymie a reçu dans le passé et reçoit encore aujourd'hui une grande attention au niveau de l'analyse des unités lexicales isolées de la langue. L'étude de ses manifestations dans le discours a cependant mobilisé un enthousiasme beaucoup plus modéré (1990, p. 29).

4 Para la elección de esta formas hemos adoptado un planteamiento onomasiológico porque era el que mejor se adaptaba a nuestros fines más inmediatos, al proporcionarnos una serie de elementos conectados, vinculados por una misma función textual o por funciones textuales próximas. 
nalmente y en pocas palabras 5 . En segundo lugar, analizaremos de manera detenida el caso del marcador en fin-marcador que posee un abundante número de empleos discursivos- y veremos si éste cuenta o no con los sinónimos que habitualmente se le atribuyen. Por último, y a partir de los resultados obtenidos, atenderemos a las dificultades que surgen a la hora de decidir si dos marcadores son o no sinónimos y mostraremos el concepto de sinominia que juzgamos más operativo en este caso.

\section{EXAMEN DE ALGUNOS MARCADORES DISCURSIVOS EN LAS OBRAS LEXICOGRÁFICAS}

Hemos seleccionado una serie de obras lexicográficas de diferente tipo. Por un lado, dos diccionarios de lengua: el Diccionario de la Real Academia española (1992) (en adelante DRAE) y el Diccionario de uso del español (1966-1967) de MARIA Moliner (en adelante DUE). Por otro, tres diccionarios específicos relativos a relaciones léxicas: el Ensayo de un diccionario español de sinónimos y antónimos (1967) de F. SAINZ DE Robles, el Diccionario de sinónimos (1975) y el Diccionario manual de sinónimos y antónimos (1991), ambos de S. GILI GAYA.

Vamos, en primer lugar, a revisar las definiciones que ofrecen los diccionarios de lengua. El DRAE (1992) da dos acepciones de en fin:

en fin

1. Finalmente, últimamente.

2. En suma, en resumidas cuentas y en pocas palabras (s.v. fin).

Resulta curioso observar las formas a las que nos remiten finalmente y últimamente:

\section{finalmente}

Últimamente, en conclusión (s.v. finalmente).

últimamente

Por último (s.v. últimamente).

5 Hemos utilizado un corpus de lengua escrita y otro de lengua oral (vid. la nómina de fuentes). Los testimonios de lengua escrita provienen de artículos periodísticos, libros de texto y obras de carácter literario. Los de lengua oral proceden del corpus de conversación semidirigida que se está elaborando en estos momentos en la Universidad de A Coruña. Este corpus de lengua hablada, dirigido por el Prof. Mauro Fernández Rodríguez, ha sido financiado por la Universidad de A Coruña, la DGICYT (PB90-0324) y la Xunta de Galicia (XUGA10402A90). 
Según la información que nos proporciona el $D R A E$ (1992), en fin es sinónimo de finalmente pero finalmente no tiene como sinónimo a en fin y lo mismo sucede con últimamente. Además, estas formas nos llevan a otras dos -en conclusión y por últimoque no estaban entre las acepciones de en fin. Por otro lado, sobre la voz en conclusión dice:

en conclusión

En suma, por último, finalmente (s.v. conclusión).

Y esta forma nos remite al segundo grupo de acepciones:

en suma

En resumen (s.v. suma).

en resumidas cuentas

En conclusión o con brevedad (s.v. cuenta).

en dos, o en pocas, palabras, en una palabra

Expres. figs. con lo que se significa la brevedad o conclusión con que se expresa o dice una cosa (s.v. palabra).

Por su parte, el DuE (1966-1967) ofrece un tipo de definición semejante a la última que hemos visto del $D R A E$. Las definiciones perifrásticas intentan explicar el(los) posible(s) sentido(s) de la forma en cuestión. Tienen la ventaja de no remitirnos directamente a otras partículas. Veamos lo que el DUE dice a propósito de en fin, en conclusión, en resumen y en suma:

en fin

Expresión con que se pasa a exponer la conclusión o resumen de lo que se viene diciendo o hablando: "En fin: que no puedo ir hoy" (s.v. fin). en conclusión

Expresión con la que se pasa a decir una consecuencia a que se ha llegado, generalmente con decepción o disgusto: "En conclusión, que no has hecho el trabajo" (s.v. conclusión).

en resumen

(expresión calificativa o adverbial). (I) De manera resumida: "Expuso en resumen las características del proyecto". (II) Se emplea para exponer la consecuencia a que se llega después de un razonamiento, una discusión, etc.: "En resumen, que no quieres venir". (III) Puede hacer también de expresión correctiva pospuesta a "aunque": "Aunque, en resumen, todo conduce a lo mismo" (s.v. resumen).

en suma

Expresión con que se introduce la exposición de una consecuencia o resumen de lo ya dicho: "En suma, que no me conviene" (s.v. suma). 
Estas definiciones no resultan tampoco excesivamente útiles. Así por ejemplo, según el $D U E$, en resumen sólo cuando funciona como 'expresión calificativa' significa 'de manera resumida'. Sin embargo, cuando funciona como marcador ('expresión adverbial') -siempre según el DUE- no posee este significado. No se le reconoce, pues, uno de los empleos discursivos principales de esta forma, esto es, la introducción de una síntesis.

Tanto en el DUE como en el DRAE, da la sensación de que conceptos como "resumen”, “conclusión”, “cierre”, "consecuencia” prácticamente no se diferencian. Y aunque están bastante próximos, no creo que se pueda identificar, por ejemplo, la idea de abreviar con la de presentar una conclusión. Así, en los libros de texto, el resumen que aparece al final de cada lección es una síntesis de lo que se dijo con anterioridad, y normalmente no se añade nada nuevo, se trata básicamente de repetir de manera condensada. En cambio, la aparición de una conclusión (o conclusiones) no tiene por qué coincidir con una versión condensada de un discurso anterior, puede(n) consistir en una serie de deducciones que se extraen del discurso previo.

Por lo que respecta a los diccionarios de sinónimos consultados, no aparece la voz en fin pero sí finalmente, últimamentey por último. Veamos a qué piezas léxicas nos remiten estas partículas.

Federico Carlos Sainz de Robles (1967) presenta los siguientes sinónimos de finalmente:

\section{finalmente}

Últimamente, supremamente, definitivamente, en definitiva, en conclusión, por último, al fin, por fin, a la postre, de vencida, pereciendo, por contra, a última hora, en último lugar, cruz y raya, sanseacabó, apaga y vámonos, ite missa est, finis coronat opus, jacabáramos! (s.v. finalmente).

Sin embargo, cuando buscamos la voz últimamente no nos remite a finalmente sino que nos ofrece la siguiente lista de sinónimos:

\section{últimamente}

En conclusión, al cabo, por último, en suma, en resolución, resueltamente, decisivamente, punto y final (s.v. finalmente).

Por su parte, los sinónimos de por último son:

\section{por último}

Por fin, al fin, al cabo, en suma, en una palabra, en último término, para terminar, para concluir, resumidamente, últimamente, finalmente (s.v. por último). 
En el Diccionario de sinónimos (1968), Samuel Gilı GaYa bajo la voz últimamente presenta los siguientes sinónimos:

\section{últimamente}

Por último, finalmente, en conclusión, en suma y en resolución (s.v. últimamente).

Años más tarde, en el Diccionario manual de sinónimos y antónimos (1991), como sinónimos de finalmente cita:

\section{finalmente}

En suma, por último, en conclusión (s.v. finalmente).

Da la sensación de que en todos estos diccionarios 6 existe una buena dosis de arbitrariedad en relación con el grupo de marcadores que estamos analizando. Siguiendo a EDDY ROULET podemos decir que sus informaciones no son satisfactorias al menos en dos aspectos:

D'une part, elles gomment les propriétés sémantiques distinctives de ces locutions, d'autre part elles n'indiquent pas ce que ces locutions, au delà de ces différences sémantiques, ont en commun pour qu'on puisse aussi aisément les substituer les unes aux autres (1987, p. 179) ${ }^{7}$.

La situación se complica cuando vamos a los diccionarios bilingües. Los que trabajamos con marcadores y consultamos estudios en otras lenguas, nos encontramos con bastantes problemas ${ }^{8}$. A menudo, solemos acudir al diccionario en busca de un equivalente castellano de la forma en cuestión. Pero las soluciones que nos da no suelen ser

6 Por lo que se refiere a los diccionarios de sinónimos, podemos decir que su utilidad se ha puesto a menudo en entredicho. Para V. LAmIQuiz "la prueba más patente de la inexistencia de sinónimos es un diccionario de sinónimos" (1974, p. 389). La posición de J. FeRnÁNDEZ SEviLLA no es tan radical. Aunque en un primer momento admite que "desde un punto de vista técnico, los diccionarios de sinónimos constituyen una aberración, pues la lingüística moderna ha dejado bien sentado que la sinonimia perfecta no existe", más adelante afirma que "sin embargo, es indudable la existencia de la sinonimia parcial en las distintas lenguas" (1974. p. 65).

7 E. Roulet se refiere al Dictionnaire du français contemporain (Paris, Larousse, 1966) y al tratamiento que en éste se le da a un grupo de locuciones adverbiales que él denomina "reformulativas".

8 En este sentido, y con respecto a las scalar particles, W. ABRAHAm señala:

Languages and contexts differ vastly in their lexical and syntactic structures when rendering any of the German scalar particles. Characteristically, there is a variety of English (or Finnish) versions corresponding to the one German scalar particles and, vice versa, there is a broad range of German scalar versions covering one single English structure. This shows that each of the "scalar structures" has its specific and unique contextual conditions. Only occasionally do these areas of semantic, syntactic and pragmatic conditions overlap complety (1991, pp. 3-4). 
de gran ayuda. Por no poner más que un ejemplo, el diccionario Larousse español-francés / francés-español (1987) bajo la voz enfin nos remite a formas tan variadas como: 'por último, al fin, por fin, en una palabra, en fin, es decir, para abreviar' (s.v. enfin). En cambio, los equivalentes de la forma castellana en fin son 'enfin y bref (s.v. en fin). Poco nos va a aclarar la consulta de este diccionario si nos encontramos con uno de los empleos discursivos de enfin que indican A. CADIOT et al., el enfin de protesta (enfin de protestation): "Un exemple élémentaire de cet emploi: un professeur, entrant dans une salle de classe où les élèves font trop de bruit, peut essayer de rétablir le silence au moyen d'un 'en fin!'” (1985, p. 220).

\section{EL CASO DE EN FIN}

Cuando se estudian los marcadores discursivos se habla con cierta frecuencia de un valor general y de unos empleos o usos discursivos determinados. No siempre es fácil encontrar un valor general subyacente a todos los empleos discursivos de un marcador. Sin embargo, en el caso de en fin podemos hablar de un valor general: "conferir al enunciado que introduce la posibilidad de poner fin a lo que precede". Éste será el valor subyacente a todos sus usos discursivos, que, como señalábamos al principio, son varios. De ahí que podamos decir que este marcador se caracteriza por su multifuncionalidad.

En este apartado nos vamos a centrar especialmente en las acepciones que de en fin da el DRAE (1992). Recordemos que, según el DRAE, en ocasiones se podrá sustituir por finalmente, en otras por últimamente. Cuando ninguna de estas dos formas se adapta al contexto en cuestión podremos echar mano de en suma, en resumidas cuentas y en pocas palabras. Vamos a prescindir de la forma últimamente, ya que no puede funcionar como marcador, es decir, no está capacitada para establecer vínculos entre enunciados. Por tanto, estudiaremos en fin en relación con las restantes formas: finalmente, en suma, en resumidas cuentas y en pocas palabras.

A pesar de que el DRAE (1992) considera estas partículas sinónimas, intentaremos mostrar a través de testimonios concretos que no siempre son intercambiables. Así, por ejemplo, veamos qué sucede en (1):

$$
\begin{aligned}
& \text { - ¿Qué ocurre? } \\
& \text { - iQue me han robado! Llevaba cincuenta mil pesetas en billetes grandes... y no } \\
& \text { están. ¿Dónde habrá sido? ¡Claro! En las escaleras de El Corte Inglés; un mu- } \\
& \text { chacho se me echó encima y me metía pierna, pero yo pensé que iba con buenas } \\
& \text { intenciones... En fin: tendré que pasar por casa para coger dinero... (Entreme- } \\
& \text { ses variados (en adelante EV). p. 71). }
\end{aligned}
$$

¿Tendría el enunciado el mismo sentido si en lugar de en fin apareciese otro de los marcadores que cita el DRAE? Creemos que no sería válida esta sustitución: 
(1a) Finalmente: tendré que pasar por casa para coger dinero...

(1b) En suma: tendré que pasar por casa para coger dinero...

(1c) En resumidas cuentas: tendré que pasar por casa para coger dinero...

(1d) En pocas palabras: tendré que pasar por casa para coger dinero...

En (1a), (1b), (1c) y (1d) el enunciado que sigue al marcador se interpretaría de manera diferente, si bien el que más parece aproximarse al sentido de en fin es (1c).

Para realizar este análisis nos hemos centrado en los siete empleos discursivos que hemos localizado para este marcador ${ }^{9}$ : en las enumeraciones, conclusivo o de resumen, de resignación, de connivencia, de rectificación, introductor de un cambio de tema y soporte conversacional. Por lo que se refiere a los dos primeros usos, podemos decir que hay casos en que en fin se podrá considerar sinónimo de alguna de las formas con respecto a las cuales lo estamos comparando. No podemos decir lo mismo de los cinco restantes, ya que en fin aporta una serie de matices de significado que no pueden ser expresados por otro marcador.

De todos modos, y aun admitiendo que cuando en fin aparece en las enumeraciones o presentado un resumen o una conclusión, encontramos formas que poseen su mismo sentido, lo cierto es que también se hallan algunos testimonios que plantean ciertas dificultades.

Veamos, en primer lugar, los tres primeros empleos que hemos establecido para en fin.

I. En fin en las enumeraciones, conclusivo o de resumen.

En una enumeración la partícula en fin indica el cierre de la misma, e introduce o bien el último elemento de la enumeración, o bien un elemento que incluya a los demás.

En (2) en fin introduce al último elemento de la enumeración e indica que la lista está completa. Aquí la sustitución por finalmente sería válida y no supondría un cambio en el contenido proposicional de la secuencia:

(2) En cambio, su contacto con los enfermos de los hospitales, y su descubrimiento de miserias y crueldades, constituyen un nuevo "motivo de depresión". [...] Al margen de sus estudios, Andrés descubre nuevas lacras; las que rodean a Lulú, la mujer que habrá de ocupar un puesto esencial en su vida. Y, en fin, la larga enfermedad y la muerte de su hermanito, Luis, vendrá a sumarse a todo como un hecho decisivo que le conduce al escepticismo ante la ciencia y a las más negras ideas sobre la vida (Literatura española, p. 9410).

9 Cf. N. VÁZquez VeIGA (1994-95), pp. 376-382. C. Fuentes RodRíGuez (1993) también se ha ocupado de este marcador.

10 Vid. nómina de fuentes. 
No obstante, aunque en el testimonio anterior demos por válida la sustitución por finalmente, hemos encontrado algún testimonio con finalmente, que nos plantea dudas:

Pero, al propio tiempo, tenemos situaciones políticas, económicas y sociales superiores que requieren un nuevo retrato político de España para esos cuatro años próximos y decisivos. En estos momentos nuestro socialismo en el poder tiene guerras interiores, aunque inteligentemente difuminadas, y tiene que tenerlo muy claro, buscando el respaldo popular en orden a la política económica y a la política social. Y después también el tema de la corrupción con noticias que sorprenden a la opinión pública. Finalmente, el proyecto de Europa obliga a muchas cosas (Emilıo Romero, La Voz de Galicia (en adelante LVG), 22.12.91, p. 14)

No creo que en (3) finalmente y en fin sean sinónimos. Dado que en el contexto previo se está haciendo referencia a una situación más o menos problemática, en fin puede añadir al enunciado que introduce un matiz de resignación que estaría ausente con finalmente.

En (4) la forma en fin aparece precediendo a un elemento que incluye a los demás. En este caso a la idea de "poner fin" habría que añadirle la de "abreviar", podríamos hablar de "fin" + "resumen":

(4) Visi es un encanto de chica, ya la verás. Es una chica moderna, con muy buen aire, inteligente, guapa, en fin, todo. Yo creo que la quiero mucho (La colmena (en adelante LC). p. 161).

En este último testimonio podría aceptarse una sustitución por en resumidas cuentas o quizá por un en pocas palabras. Descartamos la forma en suma, porque pertenece a un registro más culto. En cambio, en el siguiente caso, extraído del corpus de lengua oral, resulta más difícil dar por válida cualquier sustitución por alguna pieza léxica:

E1: Volviendo al tema de las cárceles, ¿hay malos tratos en las cárceles? $\mathrm{H}$ : Hay de todo, de todo; tienen las celdas de castigo que se llaman, que son... recintos sin luz, y le dan media ración de comida; no tienes mantas ni sábanas. tienes que dormir desnudo, como se dice, con la ropa puesta y, y en la oscuridad alli durante $X$ tiempo, según el delito o la falta que hagas. Ahora, otra cosa no, allí la... es decir, pegar a los presos, esas cosas, únicamente que hagan una revuelta, no, si se ponen en plan bravo pues claro, pero lo demás no, no hay... otro problema. Y después claro, eso, que la rigidez que tienen que comer a su hora, levantarse a las seis de la mañana... en fin, etc., etc. (Corpus de lengua oral, $\mathrm{H}$. $60-, b)$.

No parece compatible el resto de las formas con un "etc., etc.". Este "etc., etc.", al igual que el "todo" de (4) se pueden considerar "etiquetas genéricas" que el individuo 
utiliza cuando encuentra la enumeración que está realizando poco interesante, innecesaria, imposible... En estos casos la noción de conocimiento compartido juega un papel importante. El hablante piensa que, después de las características esbozadas, lo que vendría a continuación puede ser fácilmente inferido por el destinatario. Es el marcador en fin el que suele aparecer con más frecuencia con este tipo de unidades.

Como señalamos al principio de este apartado, el marcador en fin se utiliza también con un sentido que está muy próximo al de en conclusión y al de en resumen (o en resumidas cuentas). Aparece normalmente en posición final de un texto, y lo que se introduce es una deducción de lo dicho anteriormente o una recapitulación. Al igual que hicimos en el caso anterior, hablaremos de "fin" + "conclusión" y "fin" + "recapitulación":

En fin: nuestro socialismo hace bien en plantearse su situación y su futuro, pero esta es una asignatura que tiene grandes dificultades, si no buscara acomodación en las exigencias de los tiempos actuales (Emilo Romero, LVG, 14.12.91, p. 12).

En este caso podría practicarse la sustitución (en suma, en conclusión, etc.).

Pasemos ahora a analizar aquellos casos en los que la sustitución por otro marcador, lleva aparejada una alteración en el sentido del enunciado.

\section{En fin de resignación, connivencia, rectificación, introductor de un cam- bio de tema y soporte conversacional.}

En fin a menudo se emplea para expresar resignación y suele ir seguido de expresiones que indican resignación por parte del hablante:

(7) - Por supuesto. Increíble, ineluctable, todo eso. Nada de necrologías, viejo. En esta pieza ha bastado que me fuera un día para que pasaran las cosas más extremas. En fin, lo uno servirá de consuelo para lo otro (Rayuela (en adelante $R$ ). p. 145).

(8) iQué tiempos, hijo! Hay casi tanta corrupción biológica como política y social. En fin, que sea lo que Dios quiera. Si me pasa algo ya te tendré al corriente. Recibe entretanto un beso fuerte de tu madre. Faustina (CHumy CHúmez, LVG, 8.12.91, p. 8).

Con cierta frecuencia este empleo de en fin va seguido de un silencio que sustituye al discurso que se hubiese podido producir. El hablante juzga que para ser entendido no necesita completar su enunciado:

(9) Don Roque tiene sus reglas particulares de cartomancia.

La sota de bastos salió en tercer lugar.

- ¡Pobre Lola, lo que te espera! ¡Te compadezco, chica! En fin... (LC. p. 134). 
(10) "Esta pieza está tan desordenada", pensó, besando a Talita. Apenas dejara de llorar le pediría que lo ayudara a acomodar el cuarto. Empezó a acariciarla, a decirle cosas.

- En fin, en fin - dijo Oliveira (R, p. 250).

- ¿Cómo sigue Fina?

- Más animada; bastante más animada.

- Ya te dije que el tiempo es la mejor medicina para los desengaños.

Claro. Y eso que no han pasado más que diez días. En fin... don Niceto, ¿hace una copita de chinchón? (EV, p. 203)

Como se habrá podido observar, la sustitución no se podría aceptar en estos casos. En primer lugar, para que las otras partículas adquieran el matiz de resignación deben estar acompañadas de una pronunciación especial, pero lo que resulta decisivo es que en fin suele aparecer sin un segmento que lo siga, y los demás, en principio, no pueden aparecer solos (al menos no tenemos documentado ningún testimonio). La repetición de un marcador, como en el ejemplo (10), aún sería más extraña ( ${ }^{*} e n$ resumen, en resumen; *en conclusión, en conclusión).

Al en fin que va acompañado de expresiones del tipo "ya me comprendes", "no sigo", "ya sabes a lo que me refiero", etc., lo hemos denominado en fin de connivencia. Debido al carácter eufemístico de lo mencionado con anterioridad, se indica que no se va a continuar con el tema. En (12) la sustitución por otra partícula daría lugar a un cambio de matiz:

(12) ... Don Marciano confía en que lo comprenda; le envía sus más fervientes excusas; mañana le llamará por teléfono a la hora de costumbre. Ha tenido que abandonar el local por la puerta de servicio.

- Humillante para un subsecretario.

- Sin embargo, en sus circunstancias... Se trata de un hombre público, debe cuidar su look, ya sabe, la imagen. En fin: no creo preciso insistir sobre el tema ( $E V$, p. 151).

Cuando el hablante utiliza esta forma después de hacer una determinada afirmación, para introducir una precisión o rectificación referida a algo dicho previamente (13), tampoco es posible la sustitución por otro de los marcadores que el DRAE (1992) presenta como equivalentes ${ }^{11}$ :

(13) - Pero usted trabaja, se ve -lo acusó la clocharde.

- Oh, no. En fin, le llevaba los libros a un viejo, pero hace rato que no nos vemos (R, pp. 199-200).

11 En cambio, este empleo lo comparte con otras partículas: bueno, o sea, es decir, etc. 
Parece poco probable que finalmente, en suma, en resumidas cuentas y en pocas palabras se empleen para introducir un cambio de tema (14 y 15), indicando que se deja de lado un tema y se continúa con otro nuevo, o bien, que se retoma uno mencionado anteriormente:

(14) El último cliente, don Romualdo Cañizares Pardezuela, acababa de marcharse [...] Naturalmente, Romualdo, que es solterón, no resulta cliente apetecible dada la cortedad de sus comidas [...]. Pero justamente el 15 de mayo, festividad de San Isidro Labrador, patrono de Madrid, puso de manifiesto unas virtudes ciudadanas que le conquistaron el afecto de Sento. Almorzaban junto a una mesa tres individuos [...] En fin, como veníamos diciendo, aquella otra tarde ya se había marchado el valeroso ex sargento [...] (EV, pp. 64-66).

(15) - iAh, italiano! Ahora lo comprendo: gentil, inteligente, seductor. No me negará que los italianos son así...

- Eso dicen. Pero yo soy de Alfafar, Valencia.

- También los valencianos me encantan. Tan artistas, tan luminosos, tan apasionados...

- Cuando usted lo dice... En fin. ¿para beber? (EV, p. 68).

A diferencia del resto de las formas que el DRAE presenta como equivalentes de en fin, éste puede aparecer desprovisto de contenido semántico, aunque no pragmático. Son los casos que en que utiliza como una apoyatura conversacional. En la mayor parte de los testimonios, responde a titubeos del hablante y a un intento de ganar tiempo para pensar lo que se va a exponer a continuación, actuando como una fórmula retardataria:

(17) $\quad-\ldots$ Yo creo que aquí no hemos venido solamente porque el Dire nos trae. Era fácil quedarse en el circo con Suárez Meilán, conocemos el trabajo y nos aprecian. Pero no, había que entrar aquí. Los tres. El primer culpable soy yo, porque no quería que Talita creyera... en fin, que te dejaba de lado en este asunto para librarme de vos. Cuestión de amor propio te das cuenta ( $R$, pp. 289-290).

Las demás formas a las que nos lleva el DRAE y los demás diccionarios (finalmente, en suma, en resumen, en pocas palabras, por último...) cuando definen a en fin, tienen usos discursivos de los que carece esta partícula. Así, por no poner más que un ejemplo, sería extraño encontrar el marcador en fin realizando uno de los empleos de en resumen (recapitulación condensadora, la que normalmente figura al final de los distintos capítulos de los libros de texto) ${ }^{12}$. 


\section{REFLEXIÓN FINAL}

Generalmente, cuando las obras lexicográficas se someten a examen, no suelen salir muy bien paradas. Según J. Fernández Sevilla "es un tópico difundido -en él curiosamente suelen estar de acuerdo los lingüistas y los usuarios del diccionario- el que las definiciones en uso no están bien hechas" (1974, p. 68; vid. también G. HaENSCH et al., 1982 , p. 11). Por lo que se refiere a los marcadores discursivos, en los apartados anteriores hemos mostrado lo poco satisfactorias que resultan las definiciones que ofrecen algunos diccionarios ${ }^{13}$.

Ante una definición sinonímica, tenemos que ser especialmente precavidos, ya que, como hemos visto, a menudo se presentan como sinónimas voces que en realidad no lo son, o no lo son en todos los contextos. En este estudio hemos partido de un concepto de sinonimia restringido, en el sentido de que para que dos voces sean sinónimas exigimos no sólo identidad en el significado conceptual o descriptivo, sino también en el social o expresivo (o pragmático) ${ }^{14}$. Esta noción restringida de sinonimia resulta operativa para el estudio de los marcadores, porque nos puede llevar a establecer distinciones incluso en aquellos casos en que el sentido -o los sentidos- de dos marcadores está muy próximo.

Así las cosas, encontrar un sinónimo de un marcador va a ser especialmente difícil $^{15}$. De todos modos, consideramos que esta cuestión se debe plantear en otros términos. Como señalábamos al principio, no hay que pasar por alto el hecho de que no podemos hablar de sinonimia a nivel de palabras, sino a nivel de los sentidos que tiene cada palabra. Hay que situar el debate de la sinonimia a nivel de los empleos discursivos de cada marcador. Es absurdo, por tanto, decir que la forma en fin es sinónima de finalmente. Los que pueden ser sinónimos son algunos empleos discursivos de ambas formas. Una correcta descripción de todos los empleos discursivos de un marcador es un trabajo que requiere exhaustividad, además de obligarnos a manejar un corpus muy amplio, pero es un paso previo indispensable para dar un definición coherente de la forma en cuestión. Si no se conocen sus empleos dișcursivos, la definición que se dé será de escasa utilidad.

Hasta aquí hemos atendido a la parcela del significado, sin embargo, los rasgos definitorios de los marcadores hacen que el problema de la sinonimia vaya mucho más allá del conocido debate de si debemos atender sólo al significado conceptual o debemos te-

13 Sobre la naturaleza circular de los diccionarios, cf. HURFORD y HEASLEY (1983), p. 41.

14 Señala StepHen Ulumann en su obra Semantics, que "muy pocas palabras son completamente sinónimas en el sentido de ser intercambiables en cualquier contexto sin la más leve alteración en el significado objetivo, el tono sentimental o el valor evocativo" (1962, p. 160).

15 Pensemos que en la lingüística contemporánea se afirma con frecuencia que la completa sinonimia no existe. La defensa de la tesis contraria se encuentra, por ejemplo, en G. SALvadoR (1985). 
ner en cuenta los demás ${ }^{16}$. Estos elementos pueden ocupar diferentes posiciones dentro del enunciado, aunque no todos gozan de la misma libertad de posición. Por tanto, la prueba de la sustitución en ocasiones nos dará como resultado secuencias agramaticales. Veamos un caso.

EI DRAE (1992) define así la forma total:

Total

En suma, en resumen, en conclusión (s.v. total).

Vayamos con la prueba de la sustitución en este ejemplo construido al efecto:

Tengo fiebre, me duele la cabeza y la espalda, en resumen, estoy hecho un desastre.

Tengo fiebre, me duele la cabeza y la espalda, total, estoy hecho un desastre.

Tengo fiebre, me duele la cabeza y la espalda, estoy hecho un desastre, en resumen.

*Tengo fiebre, me duele la cabeza y la espalda, estoy hecho un desastre, total.

Vemos que, independientemente del hecho de que defendamos o no que estas dos formas tengan algún empleo discursivo idéntico, la prueba de la sustitución muestra que distribucionalmente no son "equivalentes". Por consiguiente, parece que en este caso el problema de la sinonimia nos conduce a un terreno que está fuera del ámbito puramente semántico ${ }^{17}$.

El establecimiento de distinciones entre marcadores pasa por dos niveles: nivel del significado y nivel sintáctico. En este último también entrarían las posibilidades combinatorias de cada marcador (combinación con conjunciones o con otros marcadores). Se

16 Cuando se habla de sinonimia y de marcadores del discurso, es importante tener presente que los marcadores no constituyen una determinada categoría gramatical. Como indica B. FraSER "discourse markers are not drawn from a single grammatical source, but reflect sources from throughout the lexical inventory" (1990, p. 388). Por tanto, la observación que hace GiLI GAYA según la cual "las voces sinónimas necesitan desempeñar la misma función gramatical" (1975, p. x), queda invalidada. Conjunciones, verbos, sustantivos, interjecciones, adverbios, etc. pueden ser marcadores discursivos. Si el contexto lo permite, un gerundio (resumiendo). por ejemplo, puede ser sinónimo de una locución adverbial (en resumen).

17 EDDY ROULET señala a propósito de los conectores de reformulación que "il est nécessaire de dépasser une approche étroitement lexicale ou grammaticale pour étudier la fonction de ces locutions dans l'articulation du discours" (1987, p. 184). 
hace necesario, pues, atender a diferentes planos de la descripción lingüística18. Según D. SCHIFFRIN:

Although part of the communicative force of a marker is due to the definition of the discourse slot in which it is used (which is defined by its place in one (or more) discourse components), the linguistic properties of the expressions used as markers are also resposible for its communicative effect. Both referencial (semantic) meaning and grammatical (syntactic) properties may contribute (1987, p. 317).

En última instancia, será el contexto en el cual se inscribe un marcador el que determine si éste cuenta o no con algún sinónimo ${ }^{19}$. Como señala GiL GaYA en el prólogo de su Diccionario de sinónimos:

La lengua nos ofrece un repertorio léxico de carácter genérico, susceptible de múltiples aplicaciones que sólo el contexto y la situación pueden determinar [...] Al realizarse las palabras en el habla, restringen su extensión y aumentan su comprensión o número de notas que colorean y caracterizan la significación concreta con que se usan. En la palabra, como en otros tantos aspectos de lo humano, realizarse es limitarse (1975, pp. x-xi).

Al emplear un marcador en un contexto determinado, estamos "activando" una serie de características que van a definir ese empleo concreto.

No me gustaría finalizar este trabajo sin antes mencionar una de las, a mi juicio, importantes carencias de las obras lexicográficas. Éstas apenas se ocupan de los marcadores cuyo empleo está ligado principalmente a las manifestaciones orales de la lengua. Cuando encontramos alguna referencia a estas formas, se trata normalmente de consideraciones de tipo prescriptivista. Los diccionarios se han olvidado de todo lo que no sea lengua escrita, y creemos que ya es hora de que esta tendencia cambie 20 .

La necesidad de un diccionario de partículas, en el que se tengan en cuenta las manifestaciones orales y escritas de la lengua, está fuera de dudas. La elaboración de este

18 El estudio de cada caso determina el número de parámetros que hay que observar. En este sentido dice JuAN M. GONZALEZ MARTínez:

Seremos más o menos estrictos y consideraremos uno u otro ámbito según lo demande aquel fenómeno con el que nos encontremos [...] y tendremos en cuenta aquella dimensión dentro del concepto genérico de sinonimia que más rentable nos resulte en cada caso (1988-1989, pp. 208-209).

19 En relación con esta cuestión, dice JACK S. ODELL que "a full account of the role of language in communication or discourse must explain or account for the contextual as well as the sentential dimensions of meaning" (1984, p. 124).

20 A propósito de las definiciones de los diccionarios en comparación con las que se dan en la conversación ordinaria, cf. B. LEWANDOWSKA (1991), pp. 205-206. Sobre el interés de los datos procedentes de la lengua oral en los diccionarios, vid. M. F. BaCelar do NASCIMENTO Y M. A. COElHO DA MOTA (1990). 
diccionario no parece ser una tarea sencilla. No resulta excesivamente difícil señalar los "puntos débiles de los diccionarios", lo que resulta muy complicado es ofrecer algún tipo de definición alternativa, que tenga en cuenta los distintos planos de la descripción lingüística. Un intento de este tipo lo encontramos en el excelente trabajo de L. IoRDANSKAJA (1993). En su artículo presenta la descripción lexicográfica de algunas conjunciones en el Dictionnaire explicatif et combinatoire du français contemporain. Establece tres parámetros para definirlas: "Nous distinguons trois classes de paramètres. Les paramètres des classes $\mathrm{A}$ et $\mathrm{B}$ concernent le sémantisme des conjonctions, alors que les paramètres de la classe $C$ en marquent le comportement syntaxique" (p. 161).

Consideramos que la puesta en práctica de este modelo, o de otro similar, puede producir resultados satisfactorios en nuestra lengua. El hecho de que actualmente contemos con bastantes estudios sobre determinadas partículas es una buena base para comenzar a trabajar en la creación de un diccionario de partículas.

\section{BIBLOGRAFÍA}

Abraham, Werner (1991): Discourse Particles, Amsterdam, John Benjamins.

Bacelar Do Nascimento, Ma Fernanda y Ma Antonia Coelho Da Mota (1990): "L'Intérêt des Données orales dans l'Élaboration des Dictionnaires de langue", Travaux de Linguistique, 21, pp. 71-79.

CADIOT, AnNe et al. (1985): "Enfin, marqueur metalinguistique", Journal of Pragmatics, 9, pp. 199-239.

Fernández Sevilla, Julo (1974): Problemas de lexicografía actual, Bogotá, Instituto Caro y Cuervo.

FRASER, BRUCE (1990): "Discourse Markers", Journal of Pragmatics, 14, pp. 383-395.

Fuentes Rodríguez, Catalina (1993): “Conclusivos y reformulativos", Verba, 20, pp. 171-198.

Garcla Pelayo, Ramón y Jean Testas (1987): Larousse moderno (français espagnol/español francés), Barcelona, Larousse.

Gilı GaYa, Samuel (1975): Diccionario de sinónimos, Barcelona, Bibliograf, $5^{\mathrm{a}}$ ed. [ $1^{\mathrm{a}}$ ed. 1968].

Gilı Gaya, Samuel (1991): Diccionario manual de sinónimos y antónimos, Barcelona, Vox, 8 a ed.

González Martínez, Juan Miguel (1988-1989): "La sinonimia. Problema metalingüístico", Anales de Filología Hispánica, 4, pp. 193-210.

GuTiérREz Ordóñez, SAlvador (1989): Introducción a la semántica funcional, Madrid, Síntesis.

HAENSCH, GüNTHER et al. (1982): La lexicografía. De la lingüística teórica a la lingüistica práctica, Madrid, Gredos.

Hurford, James R. y Brendan Heasley (1983): Semantics a Coursebook, Cambridge, Cambridge University Press [Traducción española manejada, Curso de semántica, Madrid, Visor, 1988]. IORDANSKAJA, LIDIJA (1993): "Pour une description lexicographique des Conjonctions du Français contemporain", Le Français Moderne, 61, pp. 159-190.

Lamiquiz, Vidal (1974): Lingüistica española, Sevilla, Universidad de Sevilla, $2^{\text {a }}$ ed. 
LeWandowska-Tomaszczyk, Barbara (1990): "Meaning, Synonymy, and Dictionary", en Jerzy Tomaszczyk y Barbara Lewandowska, eds., Meaning and Lexicography, Amsterdam, John Benjamins, pp. 181-208.

LYoNS, JOHN (1977): Semantics, Cambridge, Cambridge University Press [Traducción española manejada, Semántica, Barcelona, Teide, 1980].

Moliner, Marí (1966-1967): Diccionario de uso del español, Madrid, Gredos.

ODELL, JACK S. (1984): "Paraphrastic Criteria for Synonymy and Ambiguity", Theoretical Linguistics, 11/1-2, pp. 117-125.

Otaola Olano, Concepción (1994): Comentario y desarrollo de textos lingüísticos, Madrid, Universidad Nacional de Educación a Distancia.

PatrY, Richard y Nathan MÉnard (1990): "La Synonymie de la Langue est-elle celle du Discours? La Synonymie dans l'Analyse de la Cohésion textuelle", La Linquistique, 26/1, pp. 29-42.

ReAl ACADEMia Española (1992): Diccionario de la lengua española, Madrid, Espasa-Calpe. $21^{\text {a }}$ ed.

RoulET, EDDY (1987): "Approche pragmatique de quelques locutions adverbiales données comme synonymes par les Dictionnaires du Français contemporain", Cahiers Ferdinand de Saussure, 41, pp. 177-184.

Sainz De Robles, Federico Carlos (1967): Ensayo de un diccionario español de sinónimos y antónimos, Madrid, Aguilar.

Salvador, Gregorio (1985): "Sí hay sinónimos", en Semántica y lexicografía del español, Madrid, Paraninfo, pp. 51-56.

Schiffrin, DeBorah (1987): Discourse Markers, Cambridge, Cambridge University Press.

Ullmann, StePhen (1962): Semantics. An Introduction to the Science of Meaning. Oxford, Basil Blackwell [Traducción castellana manejada, Semántica. Introducción a la ciencia del significado, Madrid, Taurus, 1991].

VAZQUEZ VEIGA, NANCY (1994-95): "Una aproximación a algunos marcadores con función textual de 'resumen', 'conclusión' y 'cierre'", Estudios de Lingüistica, 10, pp. 349-390.

\section{NÓMINA DE FUENTES}

Cela, Camilo José (1985): La colmena, Barcelona, Noguer.

CoRTÁZAR, Julo (1985): Rayuela, Madrid, Planeta-Agostini.

Formación de un corpus de lengua hablada en la ciudad de A Coruña, Departamento de Lingüística Xeral e Teoría da Literatura, Facultade de Filoloxía, Universidade da Coruña.

La Voz de Galicia, A Coruña, 8-12-91: 14-12-91 y 22-12-91.

Tusón, Vicente y Fernando LÁzARo CARRETer (1986): Literatura española. Madrid, Anaya.

VizCAINo CASAS, FERnANDo (1991): Entremeses variados, Barcelona, Planeta. 